\title{
Pterostilbene attenuates the inflammatory reaction induced by ischemia/reperfusion in rat heart
}

\author{
MIN LV ${ }^{1}$, KEXIANG LIU ${ }^{2}$, SHAOPENG FU ${ }^{1}$, ZHE LI $^{1}$ and XIA YU ${ }^{3}$ \\ ${ }^{1}$ Department of Cardiovascular Surgery, China-Japan Union Hospital of Jilin University; ${ }^{2}$ Department of \\ Cardiovascular Surgery, The Second Hospital of Jilin University; ${ }^{3}$ Department of Pediatrics, \\ The First Hospital of Jilin University, Changchun, Jilin 130033, P.R. China
}

Received January 27, 2014; Accepted August 19, 2014

DOI: $10.3892 / \mathrm{mmr} .2014 .2719$

\begin{abstract}
The role of pterostilbene (Pte) in inflammation induced by ischemia/reperfusion is not well understood. The aim of this study was to investigate whether Pte modulates neutrophil accumulation and the induction of tumor necrosis factor- $\alpha(\mathrm{TNF}-\alpha)$ in an ischemia/reperfusion (I/R)-injured rat heart model. Rats were randomly exposed to a sham operation, myocardial ischemia/reperfusion $(\mathrm{MI} / \mathrm{R})$ alone, $\mathrm{MI} / \mathrm{R}+\mathrm{Pte}$, $\mathrm{MI} / \mathrm{R}+\mathrm{Pte}+\mathrm{L}-\mathrm{NAME}$ and $\mathrm{MI} / \mathrm{R}+\mathrm{Pte}+$ (methylene blue) MB. The results demonstrated that compared with $\mathrm{MI} / \mathrm{R}$, Pte reduced the area of myocardial infarction, the levels of myocardial myeloperoxidase, serum creatinine kinase and lactate dehydrogenase, and the production of serum and myocardial TNF- $\alpha$. These Pte-induced effects were eliminated by the administration of L-NAME, a nitric oxide (NO) synthase inhibitor, and MB, a cyclic guanosine monophosphate (cGMP) inhibitor. In conclusion, Pte produces cardioprotective and anti-inflammatory effects. These effects may be associated with an increase in NO production, the inhibition of neutrophil accumulation, and induction of TNF- $\alpha$ and cGMP signaling pathways in myocardium subjected to MI/R.
\end{abstract}

\section{Introduction}

The inflammatory reaction induced by ischemia/reperfusion (I/R) is an important process in the development of myocardial ischemia-reperfusion (MI/R) injury (1). During inflammation, various cytokines are released, including tumor necrosis factor- $\alpha$ (TNF- $\alpha$ ), interleukin (IL)- 6 and IL-8 (2). TNF- $\alpha$ triggers an inflammatory reaction in response to MI/R. In addition, vascular endothelial cell injury and inflammatory cells, such as neutrophils, which are activated by cytokines and adhesion

Correspondence to: Dr Xia Yu, Department of Pediatrics, The First Hospital of Jilin University, 71 Xinmin Street, Changchun, Jilin 130033, P.R. China

E-mail: yuxia2014@126.com

Key words: pterostilbene, ischemia/reperfusion injury, neutrophil, tumor necrosis factor- $\alpha$ molecules, are known to be involved in inflammation. Thus, levels of TNF- $\alpha$ and the degree of neutrophil infiltration may be considered to be indicators of an inflammatory reaction.

Pterostilbene (trans-3,5-dimethoxy-4-hydroxystilbene; Pte) is a naturally-derived compound found primarily in blueberries and Pterocarpus marsupium heartwood $(3,4)$. It is structurally similar to resveratrol, a compound found in red wine that has comparable antioxidant, anti-inflammatory and anticarcinogenic properties. However, Pte exhibits increased bioavailability compared with resveratrol, due to the presence of two methoxy groups, which confer properties of increased lipophilic and oral absorption (Fig. 1) (5-7). Increasing evidence suggests that Pte may have numerous preventive and therapeutic properties in a range of human diseases, including neurological, metabolic and hematologic disorders (8). Further benefits of Pte have been reported in preclinical trials, in which Pte was shown to be a potent anticancer agent in a number of malignancies (9). However, to the best of our knowledge, the role of Pte in inflammation induced by MI/R has not yet been reported. The present study therefore aimed to investigate the effect of Pte on neutrophil infiltration and TNF- $\alpha$ production in a rat model of $\mathrm{MI} / \mathrm{R}$ and its underlying mechanisms.

\section{Materials and methods}

Reagents. Pte was obtained from Sigma (St. Louis, MO, USA). The myeloperoxidase (MPO) assay kits, creatine kinase $(\mathrm{CK})$ test kits and lactate dehydrogenase (LDH) assay kits were purchased from JianCheng Bioengineering Institute (Nanjing, China). TNF- $\alpha$ enzyme-linked immunosorbent assay kits were purchased from R\&D Systems, Inc. (Minneapolis, MN, USA). L-NAME and methylene blue (MB) were purchased from Sigma-Aldrich (St. Louis, MO, USA). A bicinchoninic acid (BCA) protein quantification kit was purchased from Bio-Rad Laboratories (Hercules, CA, USA).

Animals. Fifty adult male Sprague-Dawley rats (250-300 g) were purchased from the Center of Experimental Animal in Jilin University (Changchun, China). All animals used in this study were cared for in accordance with the Guidance for the Care and Use of Laboratory Animals published by the United States National Institute of Health (NIH; publication no. 85-23, 

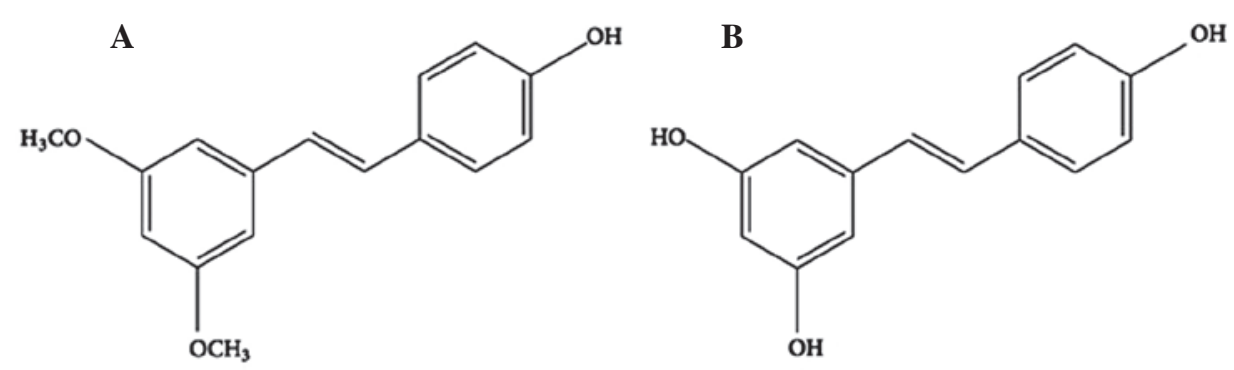

Figure 1. (A) Chemical structure of pterostilbene. (B) Chemical structure of resveratrol. In contrast to resveratrol, pterostilbene contains two methoxy groups, which increase its oral absorption and bioavailability.

revised 1996), and all procedures were approved by the Committee of Experimental Animals of Jilin University.

MI/Rmodelandexperimentalprotocol.Male Sprague-Dawley rats (weight, 250-300 g) were anesthetized intraperitoneally with $40 \mathrm{mg} / \mathrm{kg}$ sodium pentobarbital (Sigma Aldrich). Myocardial ischemia was induced by exteriorizing the heart with a left thoracic incision followed by a slipknot (5-0 silk; Johnson \& Johnson China, Ltd., Shanghai, China) around the left anterior descending (LAD) coronary artery. Following $30 \mathrm{~min}$ of ischemia, slipknots were released and animals received $120 \mathrm{~min}$ of reperfusion.

Rats were randomly assigned to five experimental groups and there were ten rats in each group. The groups were as follows: Sham group, silk was fed under the LAD coronary artery but the LAD coronary artery was not ligated; MI/R group, the LAD coronary artery was ligated for $30 \mathrm{~min}$ and then allowed $120 \mathrm{~min}$ reperfusion and was treated with vehicle [0.9\% $\mathrm{NaCl}$ intravenously (i.v.)]; $\mathrm{MI} / \mathrm{R}+$ Pte group, $100 \mu \mathrm{mol} / \mathrm{l}$ Pte i.v. was administered $5 \mathrm{~min}$ prior to reperfusion; MI/R + Pte + L-NAME group, $1 \mathrm{mmol} / \mathrm{l} \mathrm{L-NAME}$ i.v., a nitric oxide (NO) synthase inhibitor, was administered $20 \mathrm{~min}$ prior to reperfusion. At $15 \mathrm{~min}$ post-administration of L-NAME, $100 \mu \mathrm{mol} / 1$ Pte, i.v. was administered; and $\mathrm{MI} / \mathrm{R}+$ Pte + MB group, $50 \mu \mathrm{mol} / 1$ methylene blue (MB) i.v., a cyclic guanosine monophosphate (cGMP) inhibitor, was administered $20 \mathrm{~min}$ prior to reperfusion. At $15 \mathrm{~min}$ following treatment with MB, $100 \mu \mathrm{mol} / 1$ Pte, i.v. was administered.

Assay of myocardial infarct area. Following reperfusion, myocardial infarct size was determined by means of a double-staining technique and a digital imaging system (Adobe Systems Incorporated, San Jose, CA, USA) (10). Following reperfusion, the coronary blood flow was again disrupted and $4 \mathrm{ml}$ of $2 \%$ Evans blue was injected into the right ventricle in order to ensure rapid distribution around the body. Hearts were taken to a $-20^{\circ} \mathrm{C}$ refrigerator for cryopreservation. Hearts were cut into $1-\mathrm{mm}$ slices, placed in 1\% 2,3,5-triphenyltetrazolium chloride (TTC) solution, incubated for $15 \mathrm{~min}$, and maintained in $4 \%$ formaldehyde solution overnight. Evans blue-stained areas (blue staining, non-ischemic areas), TTC-stained areas (red staining, ischemic areas) and non-TTC-stained areas (white, infarct areas) were analyzed by computer using a digital imaging system. The percentage of myocardial infarct was then calculated using the following formula: Infarct area/area at risk (INF/AAR) x 100.

Determination of MPO levels. Following reperfusion, mocardial tissue was maintained at $-70^{\circ} \mathrm{C}$ for preservation. An MPO test kit was employed to detect the level of MPO in the myocardial tissue, according to the manufacturer's instructions.

Detection of CK activity. Following reperfusion, blood was taken from the carotid artery and kept at room temperature for $30 \mathrm{~min}$ at $4^{\circ} \mathrm{C}$. Serum was separated by centrifugation at $3,000 \mathrm{xg}$ for $20 \mathrm{~min}$ and maintained at $-70^{\circ} \mathrm{C}$ for preservation. A CK test kit was utilized according to the manufacturer's instructions in order to measure serum CK activity.

Determination of LDH levels. Following reperfusion, blood was taken from the carotid artery and kept at room temperature for $30 \mathrm{~min}$. Serum was separated by centrifugation at $3,000 \times \mathrm{g}$ for $20 \mathrm{~min}$ at $4^{\circ} \mathrm{C}$ and maintained at $-70^{\circ} \mathrm{C}$ for preservation. The extent of cell injury was monitored by measuring leakage of LDH. An LDH test kit was utilized according to the manufacturer's instructions in order to measure serum LDH levels.

Detection of TNF- $\alpha$ levels. Following reperfusion, the levels of TNF- $\alpha$ in myocardial tissue homogenate and serum were measured according to the manufacturer's instructions. A BCA kit was used to detect the protein quantization.

Statistical analysis. Data are presented as the mean \pm standard deviation. The significance of the differences among groups was evaluated by Student's t-test for unpaired data or Dunnett's t-test for multiple comparisons, preceded by one-way analysis of variance. SPSS version 13.0 was used for analysis (SPSS, Inc., Chicago, IL, USA) $\mathrm{P}<0.05$ was considered to indicate a statistically significant difference.

\section{Results}

Pte reduces the myocardial infarction area induced by $M I / R$. MI/R induced an area of infarction in the myocardium. Compared with the MI/R group, Pte reduced the infarcted area in the myocardium significantly. This effect of Pte was eliminated by the administration of L-NAME, a NO synthase inhibitor. In addition, the effect of Pte was 


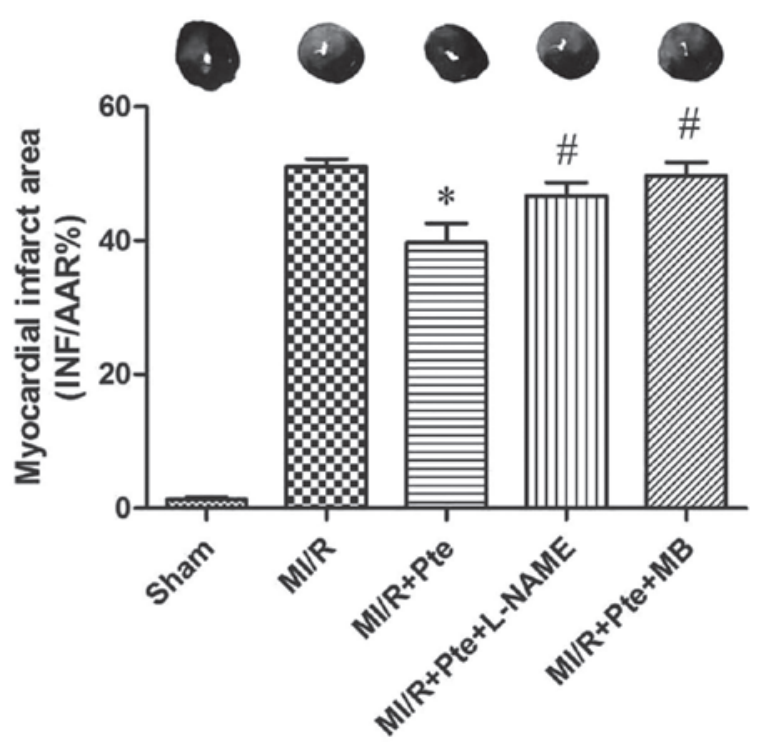

Figure 2. Comparison of myocardial infarct area (as a percentage of INF/AAR) in each group. TTC-Evans blue double staining suggested that compared with the MI/R group, Pte significantly reduced the infarct area (white area), while L-NAME and MB eliminated the effect of pterostilbene. ${ }^{*} \mathrm{P}<0.05$ compared with the MI/R group and ${ }^{\#} \mathrm{P}<0.05$, compared with the $\mathrm{MI} / \mathrm{R}+$ Pte group. $\mathrm{P}<0.0001$ between the $\mathrm{MI} / \mathrm{R}$ and sham group. INF/AAR; infarct area/area at risk; Pte, pterostilbene; MB, methylene blue; TTC, 2,3,5-triphenyltetrazolium chloride; MI/R, myocardial ischemia-reperfusion

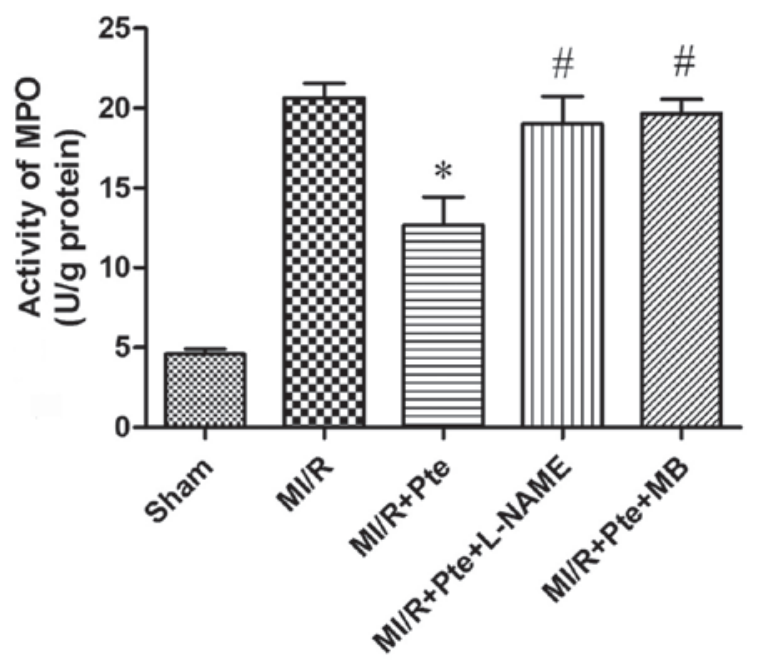

Figure 3. Comparison of MPO activity in each group. Compared with the MI/R group, the MPO activity in the MI/R + Pte group was reduced significantly. L-NAME and MB eliminated the effect of pterostilbene. ${ }^{*} \mathrm{P}<0.05$, compared with the $\mathrm{MI} / \mathrm{R}$ group and ${ }^{\#} \mathrm{P}<0.05$, compared with the $\mathrm{MI} / \mathrm{R}+\mathrm{P}$ te group. $\mathrm{P}<0.0001$ between the MI/R and sham group. Pte, pterostilbene; MB, methylene blue; MPO, myeloperoxidase; MI/R, myocardial ischemia-reperfusion.

significantly attenuated by administration of MB, a cGMP inhibitor (Fig. 2).

Pte inhibits neutrophil infiltration in MI/R tissue. Neutrophils contain a certain quantity of MPO, which accounts for $\sim 5 \%$ of dry cell weight. Thus, the activity of MPO in the myocardium may be considered as an indication of neutrophil infiltration. As shown in Fig. 3, the activity in the sham group was relatively low, whereas, by comparison, the MPO activity in the MI/R

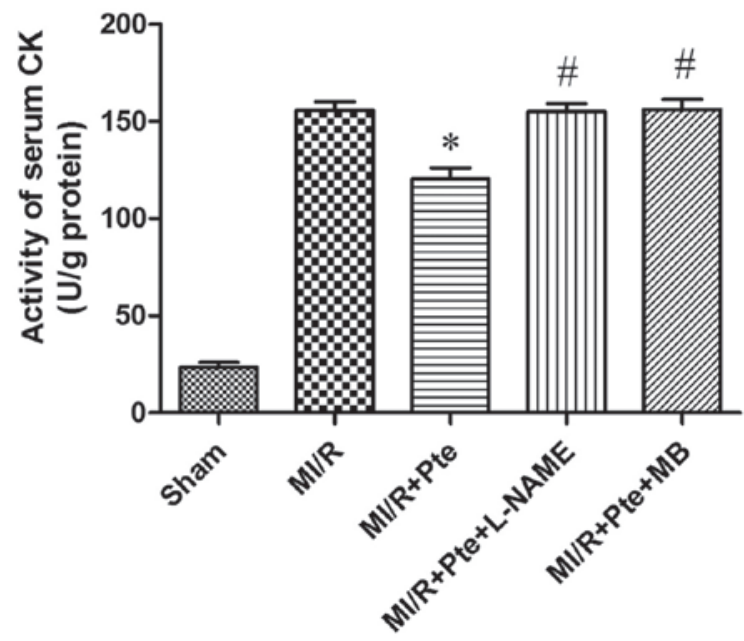

Figure 4. Comparison of serum CK activity in each group. Compared with the MI/R group, Pte reduced the serum CK activity significantly. However, this effect was eliminated by L-NAME and MB. ${ }^{*} \mathrm{P}<0.05$, compared with the $\mathrm{MI} / \mathrm{R}$ group and ${ }^{\#} \mathrm{P}<0.05$, compared with the $\mathrm{MI} / \mathrm{R}+$ Pte group. $\mathrm{P}<0.0001$ between the $\mathrm{MI} / \mathrm{R}$ and sham group. Pte, pterostilbene; $\mathrm{MB}$, methylene blue; $\mathrm{CK}$, creatine kinase; MI/R, myocardial ischemia-reperfusion.

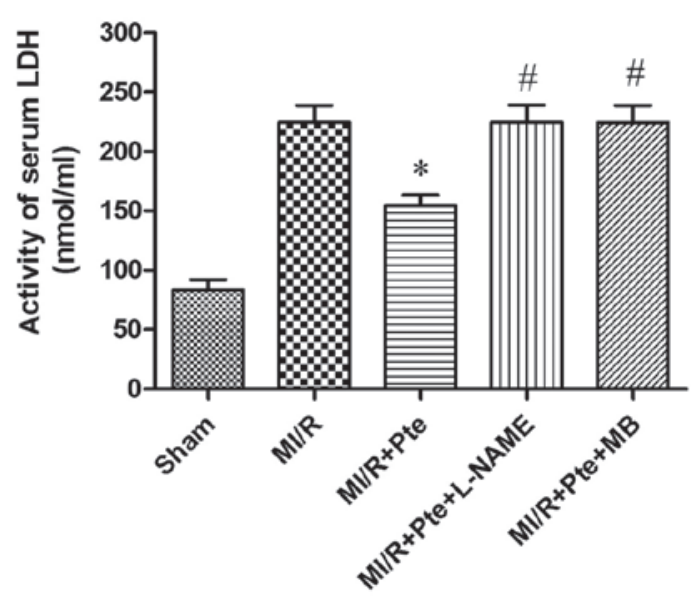

Figure 5. Comparison of serum LDH activity in each group. Compared with MI/R group, Pte reduced the serum LDH activity significantly. However, this effect was eliminated by L-NAME and MB. ${ }^{*} \mathrm{P}<0.05$, compared with the $\mathrm{MI} / \mathrm{R}$ group and ${ }^{\#} \mathrm{P}<0.05$, compared with the $\mathrm{MI} / \mathrm{R}+\mathrm{P}$ te group. $\mathrm{P}<0.0001$ between the MI/R and sham group. Pte, pterostilbene; MB, methylene blue; $\mathrm{LDH}$, lactate dehydrogenase; $\mathrm{MI} / \mathrm{R}$, myocardial ischemia-reperfusion.

group was significantly increased. Pte significantly decreased myocardial MPO activity compared with the MI/R group, whilst the administration of L-NAME and MB attenuated this effect of Pte.

Pte reduces the activity of serum $C K$ in $M I / R$ rats. As shown in Fig. 4, the activity of CK increased significantly in the MI/R group compared with the sham group. $\mathrm{CK}$ activity was significantly reduced in the $\mathrm{MI} / \mathrm{R}+$ Pte group compared with the $\mathrm{MI} / \mathrm{R}$ group. This effect of Pte was eliminated by the administration of L-NAME and MB.

Pte reduces $L D H$ activity in MI/R rats. As shown in Fig. 5, the LDH activity increased significantly in the MI/R group 
A

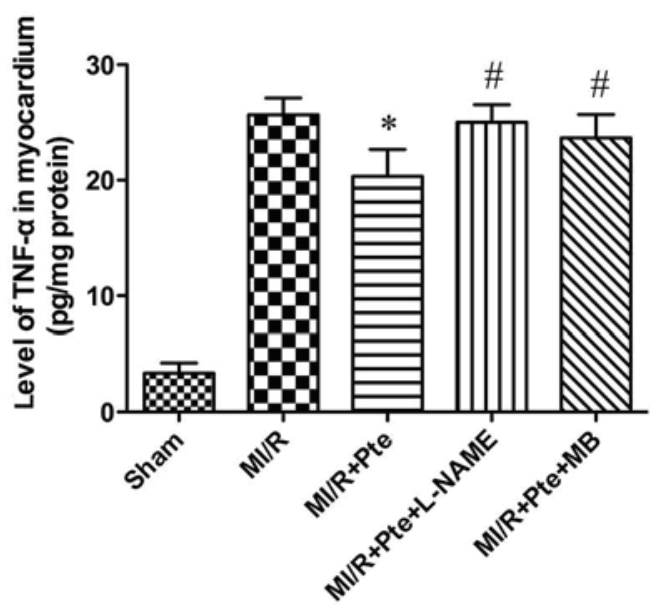

B

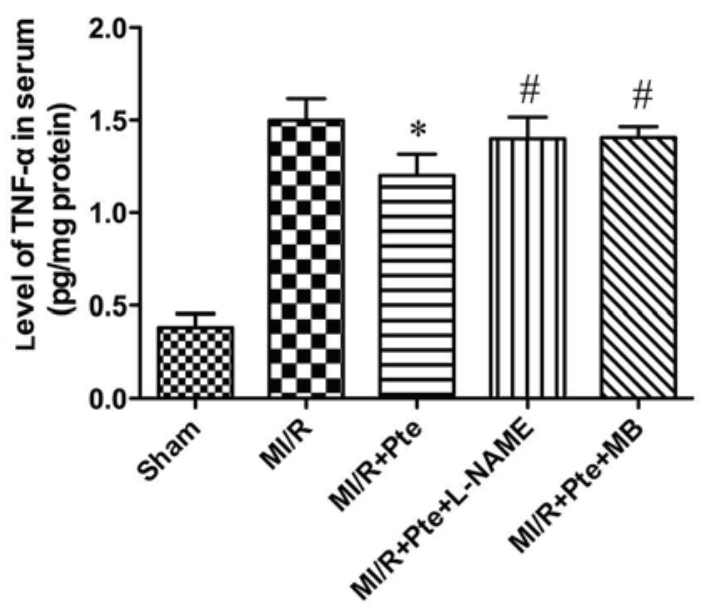

Figure 6. Comparison of levels of TNF- $\alpha$ in (A) myocardium and (B) serum. Compared with the MI/R group, pterostilbene reduced TNF- $\alpha$ levels significantly. L-NAME and MB eliminated the effect of Pte. "P<0.05, compared with the MI/R group and "P<0.05, compared with the MI/R + Pte group. Pte, pterostilbene; $\mathrm{MB}$, methylene blue; TNF- $\alpha$, tumor necrosis factor- $\alpha$; MI/R, myocardial ischemia-reperfusion.

compared with the sham group. LDH activity was significantly decreased in the $\mathrm{MI} / \mathrm{R}+$ Pte group compared with the $\mathrm{MI} / \mathrm{R}$ group. This effect of Pte was eradicated by L-NAME and MB administration.

Pte reduces TNF- $\alpha$ levels in the serum and MI/R tissue. MI/R injury is known to result in the production of increased levels of TNF- $\alpha$. Thus, myocardial and serum TNF- $\alpha$ levels were examined. As shown in Fig. 6, compared with the MI/R group, Pte significantly reduced the levels of TNF- $\alpha$ in myocardium and serum. This effect was eliminated by L-NAME and MB administration.

\section{Discussion}

The current study found that Pte reduces the inflammatory reaction induced by I/R injury by inhibiting neutrophil infiltration and TNF- $\alpha$ production. In addition, it demonstrated that NO and cGMP may be important mediators of the protective effects of Pte.

The inflammatory reaction is known to be involved in MI/R injury (1). The release of inflammatory cytokines and the aggregation and infiltration of inflammatory cells are key steps in inflammation (11).

TNF- $\alpha$ is secreted predominantly by macrophages. It promotes an inflammatory cascade by increasing the release of other proinflammatory cytokines and influencing neutrophil recruitment (12). TNF- $\alpha$ is an important cytokine in the process of inflammation, and is involved in the initiation of the inflammation induced by MI/R (13). TNF- $\alpha$ induces the release of other inflammatory mediators, increases the expression of cell adhesion factors and promotes neutrophil adhesion to endothelial cells. In addition, TNF- $\alpha$ has a negative inotropic effect, which inhibits myocardial contractility and lowers blood pressure. TNF- $\alpha$ also induces cardiomyocyte apoptosis and participates in ventricular remodeling (14). Previous studies have suggested that the level of TNF- $\alpha$ increases significantly following MI/R (15), whilst the administration of a TNF- $\alpha$ monoclonal antibody attenuated edema and aided recovery of cardiac function (16).

$\mathrm{MI} / \mathrm{R}$ injury appears to be induced in part by neutrophil activation. There are a number of mechanisms underlying this effect. Cell damage, caused by the release of oxygen free radicals, proteolytic enzymes and cytotoxic substances leads to increased neutrophil activation. In addition, inflammatory mediators cause vascular endothelial cell damage, increased vascular permeability and edema. Further activation of inflammatory cells leads to further increases in the inflammatory response (17). Finally, neutrophil adhesion to the vascular endothelium and the occlusion of small blood vessels result in a no-reflow phenomenon.

Previous studies have demonstrated an association between neutrophil and MI/R injury. The removal of neutrophils or drug-induced inhibition of neutrophil activity has been shown to reduce MI/R injury $(18,19)$. The present study found that neutrophil accumulation and TNF- $\alpha$ production increased significantly in the $\mathrm{MI} / \mathrm{R}$ group compared with the sham control group. Pte was shown to reduce neutrophil accumulation and TNF- $\alpha$ production, indicating that Pte inhibits neutrophil accumulation and TNF- $\alpha$ production and thereby attenuates neutrophil-mediated I/R injury.

It is hypothesized that NO production is associated MI/R-induced inflammation (20). Endothelial-derived NO inhibits cell adhesion factors, including P-selectin and ICAM-1 levels, thereby inhibiting leukocyte adhesion and inward membrane migration (21). Endothelial-derived NO also inhibits expression of TNF- $\alpha$ and other pro-inflammatory factors. In addition, it increases levels of IL-10 and other anti-inflammatory factors and indirectly inhibits inflammatory cells from aggregating in areas of local inflammation, thereby reducing the inflammatory response (22). In the present study, following addition of L-NAME, a NO synthase inhibitor, the protective effect of Pte was eliminated. This suggested that NO is pivotal in mediating the protective effects of Pte. Similarly, following addition of MB, a cGMP inhibitor, the protective effects of Pte were eradicated, 
indicating that the cGMP pathway is also involved in the protective actions of Pte.

In conclusion, the present study demonstrated that Pte attenuates inflammation induced by MI/R injury. The protective effects of Pte are associated with inhibition of neutrophil infiltration and TNF- $\alpha$ production, increases in the levels of NO and possible upregulation of the cGMP signaling pathway. The present study provides new insights into the mechanisms involved in the cardioprotective effect of pterostilbene against myocardial ischemia/reperfusion injury, which may be a new clinical therapy for myocardial ischemia/reperfusion injury.

\section{References}

1. Xiong J, Xue FS, Yuan YJ, Wang Q, Liao X and Wang WL: Cholinergic anti-inflammatory pathway: a possible approach to protect against myocardial ischemia reperfusion injury. Chin Med J (Engl) 123: 2720-2726, 2010.

2. Naidu BV, Farivar AS, Woolley SM, Grainger D, Verrier ED and Mulligan MS: Novel broad-spectrum chemokine inhibitor protects against lung ischemia-reperfusion injury. J Heart Lung Transplant 23: 128-134, 2004.

3. Roupe KA, Remsberg CM, Yáñez JA and Davies NM: Pharmacometrics of stilbenes: seguing towards the clinic. Curr Clin Pharmacol 1: 81-101, 2006.

4. Lin HS, Yue BD and Ho PC: Determination of pterostilbene in rat plasma by a simple HPLC-UV method and its application in pre-clinical pharmacokinetic study. Biomed Chromatogr 23: $1308-1315,2009$

5. Kapetanovic IM, Muzzio M, Huang Z, Thompson TN and McCormick DL: Pharmacokinetics, oral bioavailability, and metabolic profile of resveratrol and its dimethylether analog, pterostilbene, in rats. Cancer Chemother Pharmacol 68: 593-601, 2011.

6. Perecko T, Drabikova K, Rackova L, et al: Molecular targets of the natural antioxidant pterostilbene: effect on protein kinase $\mathrm{C}$, caspase-3 and apoptosis in human neutrophils in vitro. Neuro Endocrinol Lett 31 (Suppl 2): 84-90, 2010.

7. Athar M, Back JH, Tang X, et al: Resveratrol: a review of preclinical studies for human cancer prevention. Toxicol Appl Pharmacol 224: 274-283, 2007.

8. McCormack D and McFadden D: A review of pterostilbene antioxidant activity and disease modification. Oxid Med Cell Longev 2013: 575482, 2013.
9. McCormack D and McFadden D: Pterostilbene and cancer: current review. J Surg Res 173: e53-e61, 2012.

10. Black SC and Rodger IW: Methods for studying experimental myocardial ischemic and reperfusion injury. J Pharmacol Toxicol Methods 35: 179-190, 1996.

11. Speyer CL and Ward PA: Role of endothelial chemokines and their receptors during inflammation. J Invest Surg 24: 18-27, 2011.

12. Khimenko PL, Bagby GJ, Fuseler J and Taylor AE: Tumor necrosis factor-alpha in ischemia and reperfusion injury in rat lungs. J Appl Physiol (1985) 85: 2005-2011, 1998.

13. Batista ML Jr, Rosa JC, Lopes RD, et al: Exercise training changes IL-10/TNF-alpha ratio in the skeletal muscle of post-MI rats. Cytokine 49: 102-108, 2010.

14. Zhu J, Liu M, Kennedy RH and Liu SJ: TNF-alpha-induced impairment of mitochondrial integrity and apoptosis mediated by caspase- 8 in adult ventricular myocytes. Cytokine 34 : 96-105, 2006.

15. Meldrum DR, Cleveland JC Jr, Cain BS, Meng X and Harken AH: Increased myocardial tumor necrosis factor-alpha in a crystalloid-perfused model of cardiac ischemia-reperfusion injury. Ann Thorac Surg 65: 439-443, 1998.

16. Gurevitch J, Frolkis I, Yuhas Y, et al: Anti-tumor necrosis factor-alpha improves myocardial recovery after ischemia and reperfusion. J Am Coll Cardiol 30: 1554-1561, 1997.

17. Lefer AM, Ma XL, Weyrich A and Lefer DJ: Endothelial dysfunction and neutrophil adherence as critical events in the development of reperfusion injury. Agents Actions Suppl 41: 127-135, 1993.

18. Ma XL, Lefer DJ, Lefer AM and Rothlein R: Coronary endothelial and cardiac protective effects of a monoclonal antibody to intercellular adhesion molecule-1 in myocardial ischemia and reperfusion. Circulation 86: 937-946, 1992.

19. Chandrasekar B, Smith JB and Freeman GL: Ischemia-reperfusion of rat myocardium activates nuclear factor-KappaB and induces neutrophil infiltration via lipopolysaccharide-induced CXC chemokine. Circulation 103: 2296-2302, 2001.

20. Liu P, Hock CE, Nagele R and Wong PY: Formation of nitric oxide, superoxide, and peroxynitrite in myocardial ischemia-reperfusion injury in rats. Am J Physiol 272: H2327-H2336, 1997.

21. Li J, Wu F,Zhang H, et al: Insulin inhibits leukocyte-endothelium adherence via an Akt-NO-dependent mechanism in myocardial ischemia/reperfusion. J Mol Cell Cardiol 47: 512-519, 2009.

22. Li J, Zhang H, Wu F, et al: Insulin inhibits tumor necrosis factor-alpha induction in myocardial ischemia/reperfusion: role of Akt and endothelial nitric oxide synthase phosphorylation. Crit Care Med 36: 1551-1558, 2008. 